\title{
Synthesis \& Characterization Of Azo Compounds Containing Disubstituted Phenolic Moities
}

\author{
Sonali Lokhande ${ }^{1}$ and Nandkishore Thakare ${ }^{2}$ \\ ${ }^{1 .}$ Research Student, Department, M. S. P. Arts, Science \& K. P. T. Commerce College, \\ Manora, Dist- Washim, Maharashtra, India \\ 2. Research Guide, Department, M. S. P. Arts, Science \& K. P. T. Commerce College, \\ Manora, Dist- Washim, Maharashtra, India
}

\begin{abstract}
Dyes are organic compounds which gives colour to a substrate. Azo dyes are the largest group of colorants used in industry. Due to their ability to absorb visible light \& ease of synthesis dyes have wider industrial application and use such as textiles, fiber, leather, print and painting, also in agriculture, cosmetics \& in laboratories. In this research eight azo compounds are prepared by diazo coupling. The diazotizonium salt is formed when $\mathrm{NaNO}_{2}$ \& concentrated $\mathrm{HCl}$ is treated with substituted aromatic amines viz. Aniline, Anthranilic acid, $\alpha$-Naphathalamine, Benzidene, oNitroaniline, p-nitroaniline, sulphanilic acid and ptoulidine. The formation of novel dye took place with a sufficient yield with subsequent coupling of 2,4-dimethylphenol. The structures of prepared dyes are confirmed by Fourier transform Infrared \& proton nuclear magnetic resonance spectroscopy. Perkin Elmer spectrum used for IR spectra \& Bruker Avance $400 \mathrm{MHz}$ NMR spectrometer for NMR spectra. The IR spectra showed $3000-3500 \mathrm{~cm}^{-1}$ streching for hydroxyl group \& $1405-1550 \mathrm{~cm}^{-1}$ streching for $-\mathrm{N}=\mathrm{N}-$ bond and mass of azo compound found to be $\mathrm{m} / \mathrm{z} 304(\mathrm{M}+)$.
\end{abstract}

Keywords : Azo Dyes, diazo-coupling, 2,4dimethylphenol

\section{Introduction}

Most of the azo dye are colourful itself due to presence of $-\mathrm{N}=\mathrm{N}-$ azo group, the aromatic azo groups are highly coloured \& are often used as dyes. ${ }^{1}$ Azo dyes are the largest group of colourant with the variety of colours, so that it is largely used in textile, dyeing \& paper printing. ${ }^{2}$ In chemical industry aromatic azo compounds is used as main component for food additives, indicators, therapeutic agents, in dyes \& pigments. ${ }^{3}$ Azo dyes, shows wide application in many fields like dyeing textile fibres, biomedical studies, advanced applications in organic synthesis \& high technology areas like lasers, liquid crystalline displays, electro-optical devices \& ink-jet printers. ${ }^{4-5}$ Some azo dye possess medicinal properties like antiseptic, antiprotzoal, antifungal, antibacterial and dyes antipyretic properties. ${ }^{6-9}$

Azo dyes are the largest \& most important class of organic dyestuffs. These are the compounds consisting of a $-\mathrm{N}=\mathrm{N}$ - linkage connected to phenyl ring $^{10}$. In this compounds, $-\mathrm{N}=\mathrm{N}$ - bond acts as Chromophore. These compounds are derived by coupling $\mathrm{Ar}-\mathrm{N}=\mathrm{N}-$ salt with $\mathrm{C}_{6} \mathrm{H}_{5} \mathrm{OH}$ or amino group. The process of conversion of primary aromatic amino compounds into a diazonium salt is known as diazotization. ${ }^{11}$ The process is carried out in presence of acid \& at a very low temperature. Aromatic azo groups are formed by a coupled reaction between a Ar- $\mathrm{N}=\mathrm{N}-$ salt $\&$ a coupling agent. $^{12}$

\section{Objectives:}

1. To synthesis azo dyes from 2,4-dimethylphenol from substituted phenyl amines .

2. To study the spectral investigation of azo dyes synthesized from 2,4-dimethylphenol from aromatic amines .

\section{Materials \& Method:}

Materials :

The reagents utilized in this research are of synthetic grade. The structural composition of prepared dyes identified through $\mathrm{H}^{1}$-NMR and IR spectra. IR spectra found out with the help of Perkin Elmer spectrum FTIR instrument, the $\mathrm{H}^{1}$-NMR spectra estimate by Brucer Avance $400 \mathrm{MHz}$ NMR 
spectrometer. The crude products were recrystallize by using ethanol as solvent.

General procedure for synthesis of azo dyes containing 2,4-dimethylphenol moities :

Method: Aromatic amine like o-nitroaniline (0.01mole) was mixed with concentrated $\mathrm{HCl}(2.5$ $\mathrm{ml})$. The resultant suspension crushed into ice $\& 2.5$ ml (4N) cold $\mathrm{NaNO}_{2}$ was mixed with constant stirring. The temperature of reaction was maintained upto $0-5^{0} \mathrm{C}$, diazotization was carried out over $15-20$ mints. The diazonium salt prepared above is mixed dropwise to the Disubstituted Phenol by maintain the $\mathrm{P}^{\mathrm{H}}$ above 7 . Stired the reaction mixture \& maintained the $5-10^{\circ} \mathrm{C}$. The coloured products obtained is filtered \& dried \& recrystallized by using ethanol. The aromatic amines used in the present study are Aniline, Anthranilic acid, o-Nitroaniline, mNitroaniline, p-Toulidine, Sulphanilic acid, $\alpha$ Naphthalamine \& Benzedine.

\section{Result :}

\section{COMPOUND 1a:}

4-Hydroxy-3-Methyl-5-[(Benzenyl-1-diaze]

toluene, $\left(\mathrm{C}_{\mathbf{1 4}} \mathrm{H}_{\mathbf{1 4}} \mathrm{N}_{\mathbf{2}} \mathrm{O}\right.$ )

Molecular weight-226, Colour- Brown, Yield 64\%, IR ( $\left.\mathrm{cm}^{-1}\right)$ : 3476 (Phenolic -OH) , 1411 (vCH3), $1502(v-\mathrm{N}=\mathrm{N}-), 1243(v \mathrm{C}-\mathrm{N})$ 2922(aromatic $v \mathrm{C}-\mathrm{H})$, $1600(v \mathrm{C}=\mathrm{C})$,

$\mathrm{H}^{1} \mathrm{NMR}(\partial$ in ppm) : 2.5109( $\mathrm{s} \mathrm{H}$ ), 12.4838 ( $\mathrm{m} \mathrm{H}$ of phenolic $\mathrm{OH}$ ), 7.7469 ( s, 1H Ar-H ), 7.5333 (d $1 \mathrm{H} \mathrm{Ar} \mathrm{H}$ ), 7,2422 (d 1H Ar H) 6.6820 ( s 3H of $\mathrm{CH}_{3}$ )

\section{COMPOUND 1b:}

4-Hydroxy-3-Methyl[2nitro phenyl) diaze ] toluene, $\left(\mathrm{C}_{14} \mathrm{H}_{13} \mathrm{~N}_{3} \mathrm{O}_{3}\right)$

Molecular weight- 271, Colour- Dark Red, Yield $92 \%$, IR $\left(\mathrm{cm}^{-1}\right): 3416(\mathrm{vOH}), 1432(\mathrm{vCH} 3), 1585(\mathrm{v}-$ $\mathrm{N}=\mathrm{N}-), 1243(\mathrm{vC}-\mathrm{N}), \operatorname{aromatic}(v \mathrm{C}-\mathrm{H}) 2922,1600$ $(v \mathrm{C}=\mathrm{C}), 1504\left(v \mathrm{NO}_{2}\right)$

$\mathrm{H}^{1} \mathrm{NMR}(\partial \mathrm{in} \mathrm{ppm}): 11.3198$ (s $1 \mathrm{H}$ of phenolic $\mathrm{OH}$ ) , 8.1439 ( d 1H of Ar H), 8.0573 ( d 1H of Ar H), 7.8862( t 1H Ar H), 7.7627 ( t 1H Ar H)

\section{COMPOUND 1c:}

4-Hydroxy-3-Methyl-5-[(4-methyl )diaze]toluene, $\left(\mathrm{C}_{15} \mathrm{H}_{16} \mathrm{~N}_{2} \mathrm{O}\right)$

Molecular weight- 270, Colour-Orange, Yield - 78 $\%$,

IR $\left(\mathrm{cm}^{-1}\right): 3414(\mathrm{vOH}), 1424\left(-\mathrm{vCH}_{3}\right), 1500(v \mathrm{~N}=\mathrm{N})$, 1268(vC-N), $2915(v \mathrm{C}-\mathrm{H}$ aromatic), $1600(\mathrm{vC}=\mathrm{C})$

$\mathrm{H}^{1} \mathrm{NMR}(\partial$ in ppm) : 11.6633 (s $1 \mathrm{H}$ of $\mathrm{OH}), 7.9091$, 7.8894, 7.3837 ( d 4H of unsy $\mathrm{CH}$ ), 7.4729, 7.4033, 7.1452( s H of CH3)

\section{COMPOUND 1d:}

4-Hydroxy-3-Methyl-5-[(Naphthalene-1-

)diazeyl]toluene, ( $\mathrm{C}_{18} \mathrm{H}_{16} \mathbf{N}_{\mathbf{2}} \mathrm{O}$ )

Molecular weight- 276, Colour- Chochlate, Yield $80 \%$,

IR $\left(\mathrm{cm}^{-1}\right): 3413(\mathrm{vOH}), 1479\left(\mathrm{vCH}_{3}\right),-1507(v-\mathrm{N}=\mathrm{N}-)$, 1271(v-C-N-), $1612(v \mathrm{C}=\mathrm{C}), 2915(\mathrm{vC}-\mathrm{H}$ aromatic) $\mathrm{H}^{1} \mathrm{NMR}(\partial$ in ppm) : 11.0580 (s Phenolic-OH), 7.4797( $\mathrm{s} \mathrm{H}$ of $\mathrm{CH}_{3}$ ), 7.2327( s H of $\mathrm{CH}_{3}$ ), 7.4797( s $\mathrm{H}$ of $\mathrm{CH}_{3}$ ), 8.8441( t $1 \mathrm{H}$ of unsy $\left.\mathrm{CH}\right), 7.8837(\mathrm{t} 1 \mathrm{H}$ of unsy $\mathrm{CH}$ ), 8.4933,8.4749, 8.3757, 8.3551 ( $\mathrm{d} 1 \mathrm{H}$ of $\mathrm{CH})$

\section{COMPOUND 1e: 0}

4-Hydroxy-3-Methyl-5-[(4-nitrophenyl) diazeyl] toluene, $\left(\mathrm{C}_{\mathbf{1 4}} \mathrm{H}_{13} \mathrm{~N}_{\mathbf{3}}\right)$

Molecular weight- 383, Colour-Orange Red, Yield $82 \%$,

IR $\left(\mathrm{cm}^{-1}\right): 3413(\mathrm{vOH}), 1406\left(\mathrm{vCH}_{3}\right), 1523(\mathrm{vN}=\mathrm{N})$, 1280(vC-N), 1611( vC=C), $1523(v N O 2), 2915(v C-$ $\mathrm{H}$ aromatic)

$\mathrm{H}^{1} \mathrm{NMR}(\partial$ in ppm) : 11.4294 ( $\mathrm{s} \mathrm{H}$ of $\mathrm{OH}), 8.7023$ ( $\mathrm{s}$ $1 \mathrm{H}$ of $\left.\mathrm{SO}_{3} \mathrm{H}\right), 8.6814$ ( d H of $\left.\mathrm{CH}\right), 8.1529,8.1113(\mathrm{~d}$ $\mathrm{H}$ of $\mathrm{CH}), 7.6553,7.1964\left(\mathrm{~s} \mathrm{H}\right.$ of $\mathrm{CH}_{3}$ )

\section{COMPOUND 1f:}

\section{4-Hydroxy-3-Methyl-5-[(Benzidiene)}

diazeyl]toluene, $\left(\mathrm{C}_{20} \mathrm{H}_{19} \mathrm{~N}_{3} \mathrm{O}\right.$ )

Molecular weight- 317, Colour-White, Yield - $45 \%$, IR $\left(\mathrm{cm}^{-1}\right)$ : 1447 (vCH3), 3412 (vOHphenolic), 2931 (vC-Haromatic), $1677(v \mathrm{C}=\mathrm{C}), 1263(\mathrm{vC}-\mathrm{N}), 1204,($ $v \mathrm{~N}=\mathrm{N}) 1593,-v \mathrm{NH} 23379$

$\mathrm{H}^{1} \mathrm{NMR}(\partial$ in ppm) : 8.0205), 8.0022( $\mathrm{s}$ of $\mathrm{OH})$, 7.5737, 7.3212 ( $\mathrm{t} \mathrm{H}$ of $\mathrm{CH}), 7.4419\left(\mathrm{~s} \mathrm{H} \mathrm{CH}_{3}\right)$, 3.3859 (s H)

\section{COMPOUND 1g:}

\section{4-Hydroxy-3-Methyl-5-[(4-sulphonyl}

diazeyl]toluene, $\left(\mathrm{C}_{14} \mathrm{H}_{14} \mathrm{~N}_{2} \mathrm{O}_{4} \mathrm{~S}\right)$

, Molecular weight- 306, Colour-Red, Yield - $96 \%$, IR $\left(\mathrm{cm}^{-1}\right): 3411(\mathrm{vOH}), 1418\left(\mathrm{vCH}_{3}\right), 1579(v \mathrm{~N}=\mathrm{N})$, $1269(v \mathrm{C}-\mathrm{N}), \quad 1220, \quad 1621 \quad(\mathrm{vC}=\mathrm{C}), \quad 2971(\mathrm{vC}-$ Haromatic), 1130, 1188( $\left.v \mathrm{SO}_{3} \mathrm{H}\right)$

$\mathrm{H}^{1} \mathrm{NMR}(\partial$ in ppm) : 11.5954( $\mathrm{s}$ of $\mathrm{OH}), 7.6321$ 7.3994 ( $\mathrm{t} \mathrm{H}$ of $\mathrm{CH}$ ), 7.7419( $\left.\mathrm{s} \mathrm{H} \mathrm{CH}_{3}\right), 4.3859$ (s H )

\section{COMPOUND 1h :}

\section{4-Hydroxy-3-Methyl-5-[(4-benzoic} acid)diazeyl]toluene, $\left(\mathrm{C}_{15} \mathrm{H}_{14} \mathrm{~N}_{2} \mathrm{O}_{3}\right)$

Molecular weight- 278, Colour - Bargandy, Yield $72 \%$,

IR $\left(\mathrm{cm}^{-1}\right): 3413(v \mathrm{OH}), 1440\left(\left(v \mathrm{CH}_{3}\right), 1590(v\right.$ $\mathrm{N}=\mathrm{N}), 1295$ ( $v \mathrm{C}-\mathrm{N}), 1606(v \mathrm{C}=\mathrm{C}), 2914(v \mathrm{C}-\mathrm{H})$, $1668(v \mathrm{COOH})$

$\mathrm{H}^{1} \mathrm{NMR}(\partial$ in ppm) : 11.1681(s H of $\mathrm{OH}), 7.8062(\mathrm{~s}$ $1 \mathrm{H}$ of $\mathrm{COOH}), \quad 7.6765,7.6637(\mathrm{t} \quad \mathrm{H} \quad \mathrm{CH})$, 7.0924,7.4805( $\mathrm{s} \mathrm{H}$ of $\mathrm{CH}_{3}$ ) 


\section{Discussions :}

We report here the synthesis of some azo dyes from different eight aromatic amines by reacting with $\mathrm{HCl}$ \& cold $\mathrm{NaNO}_{2}$ solution by coupling with 2,4dimethyl phenol to give corresponding azo dyes ( as mentioned above in the table ) with a good yield. The IR spectra of synthesized compounds showed the absorption bands at $3400-3500 \mathrm{~cm}^{-1} \& 1500$ $1590 \mathrm{~cm}^{-1}$ for phenolic $\mathrm{OH} \&$ azo group respectively. Also the absorption band for $\mathrm{C}=\mathrm{C}$ at 1600 -

$1605 \mathrm{~cm}^{-1}$, aromatic $\mathrm{C}-\mathrm{H}$ is at $2915 \mathrm{~cm}^{-1}, \mathrm{NO}_{2}$ is at $1523 \mathrm{~cm}^{-1}$. Furthermore the ${ }^{1} \mathrm{H}-\mathrm{NMR}$ spectrum $-\mathrm{OH}$ proton is at $\partial 8.0811-11.500 \mathrm{ppm}$. Mass of the new compound i.e. $1-\mathrm{g}$ is found to be $\mathrm{m} / \mathrm{z} 304$.

\section{Conclusion:}

In this study, a series of eight novel azo dye were synthesized \& characterized by FT-IR, H-NMR \& MASS. All of the dyes showed a variety in colours $\&$ yield is sufficient. It can be concluded that eight azo dyes were successfully synthesized from 2,4dimethylphenol by diazo coupling reaction.

\section{References :}

[1] Bhagat S D, N. S.Thakare (2017), "Synthesis and spectral analysis of some new phenolic azo compounds. International Journal of chemTech research", 10, 160163

[2] S Asad, M. A. (2007), "Decolouration of textile azo dyes by newly isolated halophilic \& halotolerant bacteria. Bioresourse technolog"y, 2082-2088.

[3] Merino, E. (2011), "Syntheis of Azobenzenes : the coloured pieces of molecular materials". The Royal Society of Chemistry, 3835-3853

[4] S M Koshti, J. P. (2008), "Synthesis of Azo compounds contaning thymol moiety". Indian Journal of Chemistry, 47B, 329-331
[5] Kate, S. S. (2016), "Synthesis \& spectral Characterization of some Azo Amine Dyes". Journal of Global Biosciences, 5, 3615-3617

[6] S D Bhagat, N. S. (2017), "Synthesis, spectral characterization and antimicrobial activity" . International journal of pure \& applied research in engineering \& technology, 6, 510-516.

[7] N M Mallikarjuna, J. K. (2018), "Synthesis, spectroscopic characterization \& pharmacological studies on novel sulfamethaxazole based azo dyes". Journal of King Saud University-Science, 1-9.

[8] Bhavesh P. Dave, B. C. (2013), "Synthesis \& chracterization novel oligomeric azo dye". Journal of Chemistry \& Pharmaceutical Research, 5(3), 112-116.

[9] Jae Joon Kim, K. F. (2001), "Synthesis, structure \& UV-VIS absorption spectra of azo dyes derived from thiazole Dimers". Journal of chem society, The Royal society of Chemistry, 379-387.

[10] Aljamali, N. M. (2015), "Review in Azo Compounds \& its Biological Activity". Biochemistry \& Analytical Biochemistry, 4(2), 1-4.

[11]Chatwal, G. R. "Synthetic Organic Chemistry". Delhi: Himalaya Publishing House.

[12] K M Rathod, N. S. (2013). "Synthesis \& antimicrobial activity of Azo compounds containing m-creasol moiety". Chemical Science Transactions, 2(1), 25-28. 